\title{
Genome-wide analysis of acetivibrio cellulolyticus provides a blueprint of an elaborate cellulosome system
}

\author{
Bareket Dassa', Ilya Borovok ${ }^{2}$, Raphael Lamed ${ }^{2}$, Bernard Henrissat ${ }^{3}$, Pedro Coutinho ${ }^{3}$, Christopher L Hemme ${ }^{4}$, \\ Yue Huang ${ }^{4}$, Jizhong Zhou ${ }^{4}$ and Edward A Bayer ${ }^{1 *}$
}

\begin{abstract}
Background: Microbial degradation of plant cell walls and its conversion to sugars and other byproducts is a key step in the carbon cycle on Earth. In order to process heterogeneous plant-derived biomass, specialized anaerobic bacteria use an elaborate multi-enzyme cellulosome complex to synergistically deconstruct cellulosic substrates. The cellulosome was first discovered in the cellulolytic thermophile, Clostridium thermocellum, and much of our knowledge of this intriguing type of protein composite is based on the cellulosome of this environmentally and biotechnologically important bacterium. The recently sequenced genome of the cellulolytic mesophile, Acetivibrio cellulolyticus, allows detailed comparison of the cellulosomes of these two select cellulosome-producing bacteria.

Results: Comprehensive analysis of the A. cellulolyticus draft genome sequence revealed a very sophisticated cellulosome system. Compared to C. thermocellum, the cellulosomal architecture of A. cellulolyticus is much more extensive, whereby the genome encodes for twice the number of cohesin- and dockerin-containing proteins. The A. cellulolyticus genome has thus evolved an inflated number of 143 dockerin-containing genes, coding for multimodular proteins with distinctive catalytic and carbohydrate-binding modules that play critical roles in biomass degradation. Additionally, 41 putative cohesin modules distributed in 16 different scaffoldin proteins were identified in the genome, representing a broader diversity and modularity than those of Clostridium thermocellum. Although many of the A. cellulolyticus scaffoldins appear in unconventional modular combinations, elements of the basic structural scaffoldins are maintained in both species. In addition, both species exhibit similarly elaborate cellanchoring and cellulosome-related gene- regulatory elements.
\end{abstract}

Conclusions: This work portrays a particularly intricate, cell-surface cellulosome system in A. cellulolyticus and provides a blueprint for examining the specific roles of the various cellulosomal components in the degradation of complex carbohydrate substrates of the plant cell wall by the bacterium.

Keywords: Cellulosomics, Clostridium thermocellum, Scaffoldin, Cohesin, Dockerin

\section{Background}

Plant cell walls are composed of different types of recalcitrant polysaccharides, notably cellulose, which together with lignin form a rigid, stable composite material. Microbial degradation of these polysaccharides and its conversion to sugars is a key step in the carbon cycle, and its subsequent conversion to ethanol is a vital objective for

\footnotetext{
* Correspondence: ed.bayer@weizmann.ac.il

'Department of Biological Chemistry, The Weizmann Institute of Science,

Rehovot, Israel

Full list of author information is available at the end of the article
}

society [1]. One of the major paradigms for efficient degradation of cellulose is a supramolecular, multi-enzyme complex called the cellulosome, which was demonstrated in various bacteria [2-7]. The cellulosome harbors a multiplicity of carbohydrate-active enzymes, i.e., glycoside hydrolases (GHs), carbohydrate esterases (CEs) and polysaccharide lyases (PLs). These include multiple endoglucanases, cellobiohydrolases, xylanases and other degradative enzymes which work synergistically to attack heterogeneous, insoluble cellulose substrates [8-11]. These enzymes are very similar in their mode of action to those

\section{Biomed Central}


of the free enzyme systems of other bacteria and fungi, except that the cellulosomal enzymes contain a dockerin module in place of a carbohydrate-binding module (CBM), which would target the individual enzymes to the substrate. Scaffoldin (Sca), a major cellulosomal subunit, is responsible for organizing the cellulolytic subunits into the complex. The dockerin-borne enzyme subunits are integrated into the scaffoldin subunit via the tenacious protein-protein interaction with multiple copies of cohesin modules. The scaffoldin subunit also contains a single CBM that attaches the entire enzymatic complex (as well as the parent bacterial cell) to the cellulose substrate, thereby enabling efficient synergistic degradation of the substrate.

Acetivibrio cellulolyticus is a mesophilic, anaerobic, gram-positive bacterium, known both for its efficient degradation of crystalline cellulose [12-15] and for its distinct protuberant cell surface ultrastructure [16]. A gene cluster of four cellulosomal scaffoldin proteins (ScaA-ScaD) from A. cellulolyticus ATCC 33288 was studied during the past decade [17-19]. The primary scaffoldin, ScaA (previously termed CipV), contains a singular intrinsic family-9 glycoside hydrolase $(\mathrm{GH})$ and mediates direct incorporation of the dockerin-containing enzymes through its seven type-I cohesins. It is bound to the cell surface via its $\mathrm{C}$-terminal $\mathrm{X}$-module/dockerin dyad (XDoc) to at least two additional scaffoldins. Thus, ScaA can either interact directly with the ScaD surfaceanchoring scaffoldin or it may bind to the ScaC scaffoldin indirectly through a ScaB adaptor scaffoldin $[18,20,21]$. ScaC and ScaD serve as anchoring scaffoldins, owing to their C- terminal S-layer homology (SLH) modules, but unlike any other scaffolding yet described, the ScaD protein harbors two different types of cohesin (types I and II), which exhibit two divergent dockerinbinding specificities [19]. Thus, only four scaffoldin proteins of the bacterium have been recognized and analyzed prior to sequencing of its genome [22].

Despite the limited genomic information available at the time, a putative model of the cellulosome architecture was proposed, suggesting alternative modes of interactions among the $A$. cellulolyticus scaffoldin components and mechanisms of attachment to the cell surface. Still, the exact model and stoichiometry of the cellulosome arrangement is currently unknown. Original experiments indicated the presence of additional putative cellulosomal enzyme components [18] and scaffoldins [19] which were probed by the ScaC cohesin but were never fully identified.

The expansion of genome sequencing efforts during the past decade has also provided information regarding several cellulosome-producing bacteria [23-26], and their genome-wide comparison has spawned the field of cellulosomics [5], i.e., a general overview of cellulosome- related constituents of a given bacterium. The recent sequencing of the $A$. cellulolyticus genome [22] has thus enabled identification and analysis of numerous additional cellulosomal components, gene regulatory elements, and cell anchoring modules in the bacterium, as documented in this communication. The interrelationship of the A. cellulolyticus cellulosome components was further explored by genome-wide comparison of its cellulosomal architecture and subunits with those of Clostridium thermocellum.

\section{Results and discussion}

Multiplicity of scaffoldins and cohesin-containing proteins The Acetivibrio cellulolyticus CD2 genome [22] is the largest among the known cellulolytic bacteria (6.1 Mb). Analysis of its recent genome sequence revealed $41 \mathrm{pu}-$ tative cohesin modules, distributed in 16 scaffoldins, some of which have both cohesins and dockerins in the same polypeptide chain (Figure 1 and Additional file 1: Table S1). These include the four genes of the scaffoldin cluster (scaA, scaB, scaC and scaD), which were originally identified, sequenced and characterized in $A$. cellulolyticus ATCC 33288 [17-19].

The previous publications have indicated that this mesophilic bacterium harbors an intricate cellulosome system, which is characterized by several unique properties that distinguish $A$. cellulolyticus from the archetypical C. thermocellum cellulosome: The progression of the ScaA primary scaffoldin, the ScaB adaptor scaffoldin and the ScaC anchoring scaffoldin, with their resident cohesins (7, 4 and 3 , respectively), suggests that the resultant fully occupied cellulosome complex would include up to 84 dockerin- containing proteins (enzymes) in addition to the intrinsic ScaA cellulase. The second type of cellulosome complex comprises a divergent anchoring scaffoldin, ScaD, which contains different cohesin specificities: two type-II cohesins that incorporate two ScaA subunits with their complement of dockerin-containing enzymes and a single type-I cohesin that binds a lone dockerin-containing protein.

Comparison of the original $A$. cellulolyticus sca genes which were individually sequenced by conventional methodology [17-19] to those of the newly sequenced genome shows only a few differences (two nucleotide substitutions out of 2601 in the ScaB gene [GenBank: ZP_09464032]).

\section{Modular nature of the cohesin-containing proteins}

In the present work, the sequenced $A$. cellulolyticus genome revealed 12 cohesin- containing proteins in addition to the previously known four major scaffoldins encoded by the sca gene cluster. Figure 1 presents their modular architecture. All of the proteins listed in the figure, 


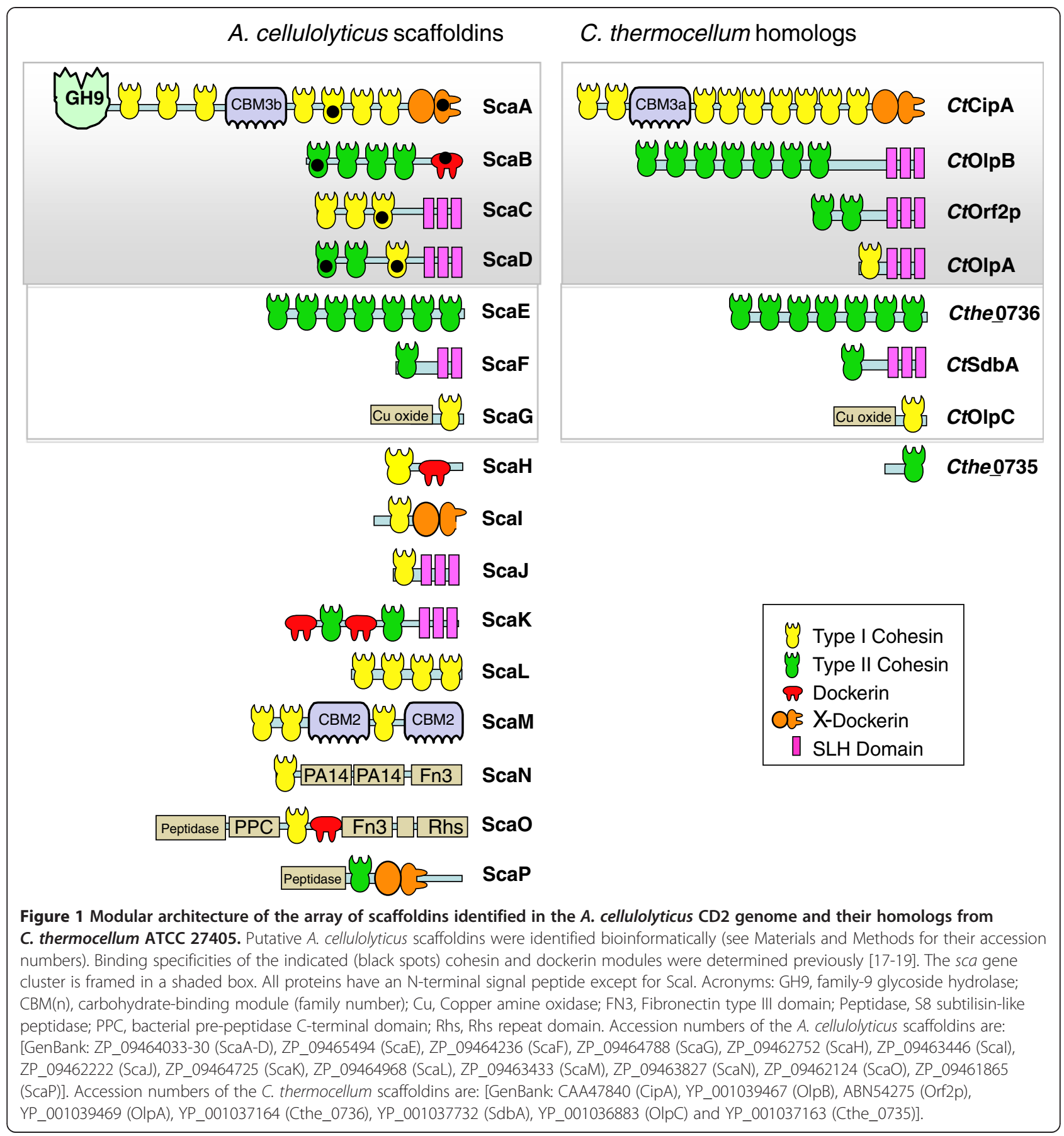

except for ScaI, contain a credible signal peptide, suggesting that these proteins would be secreted.

The cohesin modules exhibit a variety of intriguing sequence features. Like $C$. thermocellum, the 41 cohesins of A. cellulolyticus are classified into two types: type I (26 modules) and type II (15 modules). We examined the conservation of the cohesin sequences and compared copies of the various cohesin modules within a given scaffoldin protein, and among the different scaffoldins. The overall sequence similarity among the A. cellulolyticus cohesin modules ranges from 41 to $97 \%$. Some scaffoldins contain similar repeats of the same type of cohesin module, whereas others bear a single cohesin. ScaD alone contains a combination of two heterogeneous cohesin types on the same polypeptide chain. As has been experimentally documented [17-19], the cohesin type (i.e., type I or type II cohesin) does not necessarily indicate its binding specificity to a given dockerin. For example, the cohesins from ScaA and ScaC (Figure 1) are all type I according to their sequences, but they bind to different dockerins - the ScaA 
cohesins bind to the dockerin-bearing enzymes, and the $\mathrm{ScaC}$ cohesins bind to the ScaB dockerin.

The combination of S-layer homology (SLH) modules with cohesin modules on the same polypeptide suggests a role for such proteins in anchoring the cellulosome assemblies or specific enzymes to the cell wall of the gram-positive bacterium $[27,28]$.

In addition to the previously described anchoring scaffoldins, $\mathrm{ScaC}$ and $\mathrm{ScaD}$, three more proteins which contain SLH modules are now revealed, i.e. ScaF [GenBank: ZP_09464236], ScaJ [ZP_09462222] and ScaK [ZP_09464725]. Of the 37 SLH- containing proteins encoded in the A. cellulolyticus genome, ScaK was identified with an SLH module, two dockerins and two cohesin modules. This is the first example of such an architectural arrangement of a cell-surface anchoring scaffoldin that contains both types of cellulosome-related modules.

Uniquely, one cohesin-containing protein also contains two family 2 CBMs, interspacing its type-I cohesins (ScaM, [ZP_09463433]). To our knowledge, this is the first description of a scaffoldin-borne CBM2; all previous CBMs located on scaffoldins have been from family 3 . CBM2s have been described as ancillary modules of enzymes and were shown to bind efficiently to cellulose and/or xylan. Thus, their appearance on a scaffoldin may serve to enhance the substrate-binding function of the dockerin- containing enzymes, which bind to this scaffoldin protein via its type-I cohesins. Other cohesins were identified in novel types of scaffoldins which bear FN3 (Fibronectin type III) repeats, PA14 (protective antigen) domain, peptidase or other extracellular modules.

\section{Relationship between cohesins of A. Cellulolyticus and C. Thermocellum}

Complex cellulosome architectures were previously proposed for A. cellulolyticus and C. thermocellum, which are two phylogenetically related Clostridiales species, as implied from their $16 \mathrm{~S}$ rRNA analysis [29]. The C. thermocellum genome contains 8 cohesin-containing proteins (scaffoldins), whereas A. cellulolyticus has twice the number of scaffoldins. The cellulosome system of C. thermocellum was selected as the reference strain, since it is the first-identified and best-established multiple-scaffoldin system, which possesses clear similarities to that of A. cellulolyticus [4].

Interestingly, three pairs of scaffoldins from both species have the same basic modular organization. Thus, two homologous scaffoldins, A. cellulolyticus ScaE [GenBank: ZP_09465494] and C. thermocellum Cthe_0736, each consist of seven consecutive type-II cohesins (Figure 1). Likewise, ScaF [GenBank: ZP_09464236] and C. thermocellum $(\mathrm{Ct}) \mathrm{SdbA}$ have a similar architecture comprising a single type-II cohesin followed by an SLH module. Finally, ScaG [GenBank: ZP_09464788] and the cell- surface Ct OlpC [30] both possess a single type-I cohesin, following a unique domain annotated as copper amine oxidase-like [Pfam: PF07833].

It is important to examine the phylogenetic relationship among the different cohesins within and between the two species, in order to reveal clues regarding their divergence (Figure 2). For example, all seven of the A. cellulolyticus $\mathrm{ScaE}$ cohesins are similar to each other and are thus clustered together on a single branch of the phylogenetic tree. In contrast, the seven Cthe_0736 cohesins are interwoven on different branches, such that cohesins 1 and 4 are closely related, as are cohesins 5 to 7 , indicating domain duplication events in the evolution of this protein. Further diversification of Cthe_0736 is evident in the acquisition of cohesin 2 which bears similarity to divergent type-II cohesins of other $C$. thermocellum anchoring scaffoldins. The seven A. cellulolyticus ScaE cohesins appear to be most similar to Cthe_0736 cohesins 3 and $5-7$, which presumably suggests a common origin.

The cellulosomes of both species harbor several anchoring proteins, composed of one or more cohesins with SLH modules. For example, ScaF and Ct SdbA have a single type-II cohesin followed by SLH repeats. Yet, their cohesins are clustered on very different branches on the tree (Figure 2), suggesting that their parent proteins are the product of different evolutionary pathways. The ScaF cohesin is closely related to those of ScaE and the above-mentioned Cthe_0736 cohesins, whereas that of Ct SdbA is more similar to those of the other C. thermocellum anchoring scaffoldins. In a similar manner, each of the anchoring scaffoldins, ScaJ and Ct OlpA, harbors a single type-I cohesin, located on divergent branches of the phylogenetic tree. As opposed to the type-II cohesins, the relationship among type-I cohesins is more straightforward, where cohesins from each species are clustered on separate branches of the tree.

\section{Abundance of dockerins in the A. Cellulolyticus genome}

The A. cellulolyticus genome is particularly enriched with dockerin-containing genes, and 143 genes that contain putative dockerin modules were identified. Therefore, A. cellulolyticus contains almost twice the number of dockerins as other Clostridial bacteria, such as Clostridium cellulolyticum ( $>60$ dockerins) or Clostridium thermocellum ( $>70$ dockerins) [23,31,32]. Only the genome of Ruminococcus flavefaciens FD-1 is known currently to contain more dockerin-containing genes $(>220)$ [26,33]. Unlike the $R$. flavefaciens dockerins, which are classified into 6 major groups and 11 subgroups [33], the A. cellulolyticus dockerins are highly similar, with the exception of six dockerins located downstream of an $\mathrm{X}$ module. These latter dockerins 




have distinctive sequence features compared to the rest of the A. cellulolyticus dockerins. Their X-modules are of family X60 [34], which display significant sequence similarity with the $\mathrm{X}$-module at the $\mathrm{C}$-terminus of the C. thermocellum CipA scaffoldin. Indeed, several of these X-dockerin pairs are found at the C-terminus of A. cellulolyticus scaffoldins (ScaA, ScaP and ScaI). Interestingly, ScaI protein contains an X-dockerin modular dyad with a truncated type-II dockerin at its $\mathrm{C}$-terminus.
The characteristic sequence conservation profile [3537] of the A. cellulolyticus dockerin module is shown in Figure 3 . The sequence similarity among A. cellulolyticus dockerin modules is $53 \%$ on average $(73 \%$ for the most similar dockerins pairs, with no two identical dockerins). Like the dockerins in $C$. thermocellum and unlike those of $R$. flavefaciens, each A. cellulolyticus dockerin module contains two canonical $\mathrm{Ca}+2$ binding repeats, followed by putative helices and linkers. Examination of the 
A. cellulolyticus -1 (137)

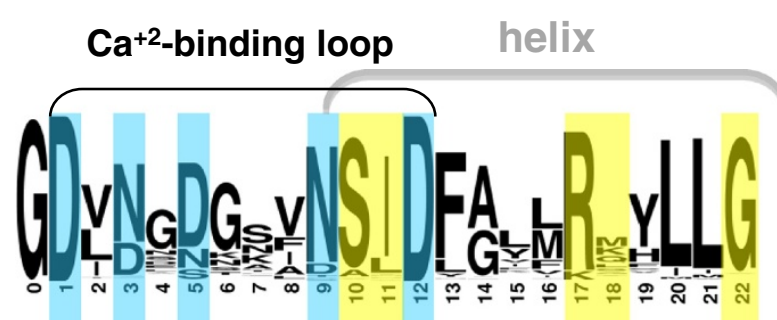

A. cellulolyticus -2 (137)

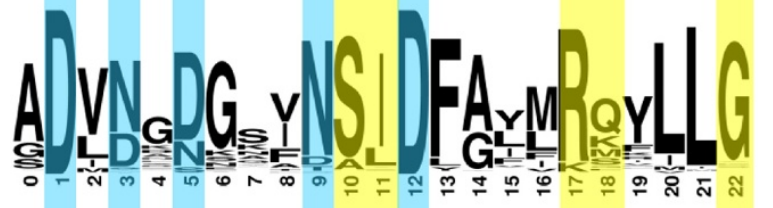

C. thermocellum -1 (71)

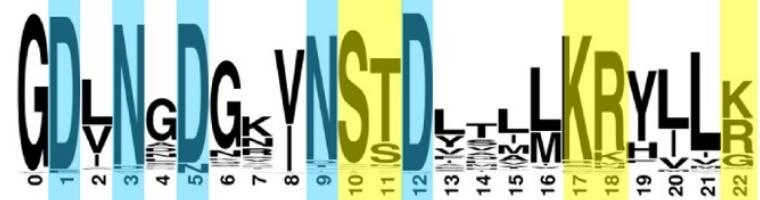

C. thermocellum -2 (71)

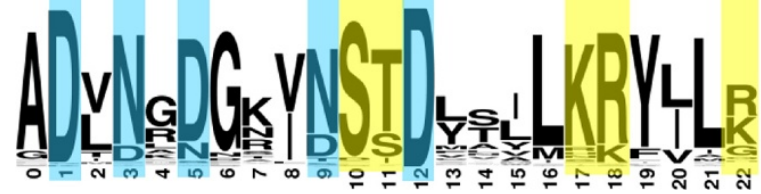

Figure 3 Sequence conservation pattern of dockerin modules. The two internal dockerin repeats of $A$. cellulolyticus (based on 137 sequences) and $C$. thermocellum (71 sequences) are represented by sequence logos. Positions of calcium binding residues are shown in cyan, and putative recognition residues are shown in yellow.

putative "recognition" residues of the dockerins, which may participate in their tight binding interface with cohesins, shows a conserved pattern of the two repeated segments wherein $\mathrm{S}(\mathrm{I} / \mathrm{L})$ residues occupy positions 10 and $11, \mathrm{R}(\mathrm{X})$ positions 17 and 18 , and a highly conserved $\mathrm{G}$ in position 22 (Figure 3, in yellow). The corresponding positions in the C.thermocellum dockerins are $S(T / S)$, $\mathrm{K}(\mathrm{R} / \mathrm{K})$ and $\mathrm{K} / \mathrm{R} / \mathrm{G}$, respectively. Position 18 is much less conserved in the A. cellulolyticus dockerins than those of $C$. thermocellum, whereas the reverse is true for position 22. Some modifications are evident in position 11 of the A. cellulolyticus dockerin sequences. For example, the ScaK scaffoldin contains an $\mathrm{N}$ - terminal dockerin with an Asn residue in position 11 of its first dockerin repeat. ScaB dockerin contains Asn residues in both repeats, and instead of the conserved Asn in position 9 it contains a positively charged Lys or Arg residue. In the case of ScaB, these modifications lead to different specificity characteristics, as the dockerin binds selectively to the cohesins of ScaC [18].

\section{Diversity of dockerin-containing enzymes}

A. cellulolyticus grows on amorphous and crystalline forms of cellulose, xylans, and cellobiose [38,39]; the bacterium can also be adapted to grow on glucose and xylose $[13,40]$. Consequently, it was presumed in these early works that the bacterium produces endoglucanases, exoglucanases, $\beta$-glucosidases and xylanase activities. Indeed, the present study reveals an intricate array of cellulolytic and hemicellulolytic enzymes in the A. cellulolyticus genome, capable of hydrolyzing diverse cellulosic substrates to reducing sugars.

The sequence features of the dockerin-containing enzymes of $A$. cellulolyticus were assessed using the following approach: (i) Like the cohesin-bearing proteins, the dockerin-containing proteins are multimodular in nature, composed of more than one type of module (catalytic, structural, etc.) and sometimes more than one repeat of the same module. The different modular types were therefore enumerated, in order to determine their general distribution among the $\mathrm{A}$. cellulolyticus proteins. (ii) Where appropriate, we distinguished between cellulosomal (i.e., those that harbor a dockerin) and non-cellulosomal (without a dockerin) proteins. (iii) We compared the A. cellulolyticus proteins with those of $C$. thermocellum.

Among the 143 dockerin-containing proteins, about half (63 proteins) contain one or more known carbohydrate-active CAZyme module(s) [41], and their composition is presented in Table 1 and Additional file 1: Table S1. Because of the multimodular nature of the proteins, some of them contain more than one type of catalytic 
Table 1 Comparative distribution of dockerin-containing CAZyme modules in A. cellulolyticus vs. C. thermocellum

\begin{tabular}{|c|c|c|c|c|c|c|c|c|c|c|c|c|c|c|c|c|c|c|c|c|c|c|c|c|c|c|c|c|c|c|c|c|}
\hline \multicolumn{2}{|c|}{ A. Glycoside Hydrolases families } & \multirow{2}{*}{$\begin{array}{l}1 \\
2\end{array}$} & \multirow{2}{*}{$\begin{array}{l}2 \\
1\end{array}$} & \multirow{2}{*}{$\begin{array}{l}3 \\
3\end{array}$} & \multirow{2}{*}{$\begin{array}{l}5 \\
16\end{array}$} & \multirow{2}{*}{$\begin{array}{l}8 \\
4\end{array}$} & \multirow{2}{*}{$\begin{array}{l}9 \\
21\end{array}$} & \multirow{2}{*}{$\begin{array}{l}10 \\
4\end{array}$} & \multirow{2}{*}{$\begin{array}{l}11 \\
1\end{array}$} & \multirow{2}{*}{$\begin{array}{l}13 \\
3\end{array}$} & \multirow{2}{*}{$\begin{array}{l}15 \\
1\end{array}$} & \multirow{2}{*}{$\begin{array}{l}16 \\
1\end{array}$} & \multirow{2}{*}{$\begin{array}{l}18 \\
5\end{array}$} & \multirow{2}{*}{\begin{tabular}{|l|}
19 \\
2
\end{tabular}} & \multirow{2}{*}{$\begin{array}{l}\mathbf{2 3} \\
2\end{array}$} & \multirow{2}{*}{$\begin{array}{l}26 \\
5\end{array}$} & \multirow{2}{*}{$\begin{array}{l}30 \\
3\end{array}$} & \multirow{2}{*}{$\begin{array}{l}39 \\
-\end{array}$} & \multirow{2}{*}{$\begin{array}{l}43 \\
4\end{array}$} & \multirow{2}{*}{$\begin{array}{l}44 \\
1\end{array}$} & \multirow{2}{*}{$\begin{array}{l}48 \\
2\end{array}$} & \multirow{2}{*}{$\begin{array}{l}51 \\
-\end{array}$} & \multirow{2}{*}{$\begin{array}{l}53 \\
1\end{array}$} & \multirow{2}{*}{$\begin{array}{l}59 \\
1\end{array}$} & \multirow{2}{*}{$\begin{array}{l}74 \\
1\end{array}$} & 778 & 819 & $94 \quad 10$ & 105 & 116 & 124 & Total \\
\hline A. cellulolyticus & Genome-wide & & & & & & & & & & & & & & & & & & & & & & & & & 11 & & 3 & 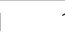 & 1 & 1 & 92 \\
\hline & Dockerin-containing proteins & - & 1 & - & 12 & 3 & 19 & 4 & 1 & - & - & 1 & - & - & - & 4 & 4 & - & 4 & 1 & 1 & -1 & 1 & 1 & 1 & -1 & & -1 & 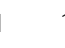 & 1 & 1 & 62 \\
\hline C. thermocellum & Genome-wide & 2 & 1 & 2 & 10 & 1 & 16 & 6 & 1 & 2 & 1 & 2 & 4 & - & 2 & 3 & 2 & 1 & 6 & 1 & 21 & 1 & 1 & -1 & 1 & -1 & & - & - & - & 1 & 73 \\
\hline & Dockerin-containing proteins & - & 1 & - & 8 & 1 & 15 & 3 & 1 & - & - & 1 & 1 & - & - & 3 & 2 & 1 & 5 & 1 & 1 & -1 & 1 & -1 & 1 & -1 & & - & - & - & 1 & 48 \\
\hline B. Polysaccharid & de Lyases families & 1 & 9 & 11 & & & & & & & & & & & & & & & & & & & & & & & & & & & & \\
\hline A. cellulolyticus & Genome-wide & 1 & 1 & 3 & & & & & & & & & & & & & & & & & & & & & & & & & & & & 5 \\
\hline & Dockerin-containing proteins & 1 & 1 & 3 & & & & & & & & & & & & & & & & & & & & & & & & & & & & 5 \\
\hline C. thermocellum & Genome-wide & 2 & 1 & 1 & & & & & & & & & & & & & & & & & & & & & & & & & & & & 4 \\
\hline & Dockerin-containing proteins & 2 & 1 & 1 & & & & & & & & & & & & & & & & & & & & & & & & & & & & 4 \\
\hline C. Carbohydrate & e Esterases families & 1 & 2 & 3 & 4 & 6 & 7 & 8 & 9 & 12 & 14 & & & & & & & & & & & & & & & & & & & & & \\
\hline A. cellulolyticus & Genome-wide & 2 & 1 & 3 & 4 & 1 & - & 1 & 1 & 7 & - & & & & & & & & & & & & & & & & & & & & & 20 \\
\hline & Dockerin-containing proteins & 2 & 1 & 3 & 1 & 1 & - & 1 & - & $4(6)$ & - & & & & & & & & & & & & & & & & & & & & & 13 \\
\hline C. thermocellum & Genome-wide & 3 & 1 & 2 & 3 & - & 1 & 1 & 1 & 2 & 1 & & & & & & & & & & & & & & & & & & & & & 15 \\
\hline & Dockerin-containing proteins & 3 & 1 & $1(2)$ & 1 & - & - & 1 & - & $1(2)$ & - & & & & & & & & & & & & & & & & & & & & & 8 \\
\hline
\end{tabular}

Numbers represent proteins which contain one or more modules of the different protein families (glycoside hydrolases, polysaccharide lyases and carbohydrate esterases) as were identified by CAZy. The number of proteins are compared between cellulosomal and non-cellulosomal (genome-wide) proteins. Data are provided for both species. Numbers of modules which appear more than once in the same protein are shown parenthetically. 
module, therefore the total sum of catalytic modules in the 63 enzymes is 80 in Table 1 (62 GH-, 13 CE- and/or 5 PLcontaining enzymes). Of the $92 \mathrm{GHs}$, about two-thirds are equipped with dockerins, suggesting that they are recruited to the cellulosome and may thus play a critical role in biomass degradation. Interestingly, the percentage of dockerin-containing GHs in the A. cellulolyticus genome is almost identical to that of $C$. thermocellum. The 62 dockerin-containing GHs belong to 19 different families according to the CAZy database (Table 1). As in all known cellulosomes produced by other species, the A. cellulolyticus cellulosome contains a single distinctive GH48 enzyme. As in C. thermocellum, the A. cellulolyticus genome also codes for a second, non-cellulosomal GH48-containing cellulase, as opposed to other characterized cellulosomeproducing species that possess only one cellulosomal enzyme. The most abundant GH family is represented by the GH9 enzymes, again like in the C. thermocellum cellulosome. This is followed by the GH5 enzymes which are also numerous in both cellulosome-producing species. Of the 21 GH9 enzymes, 10 exhibit a GH9-CBM3 motif that would potentially modulate the activity as in $C$. thermocellum and other cellulolytic bacteria [42-45]. In addition, there are three enzymes that show an extended GH9CBM3-CBM3 motif, compared to two such enzymes in $C$. thermocellum [46].

In one third of the dockerin-containing proteins (46 proteins) we identified modules which are predicted to be associated with extra-cellular proteins (i.e., FN3 modules, Leu-rich repeats, RhsA and PKD domains, see Table 2). Some of these modules are conserved in sequence, but their function is still unknown; some may represent a yet undiscovered enzyme. In this regard, a C. thermocellum dockerin-containing protein of previously unknown function was recently demonstrated to be a cellulase [47]. The dockerin-containing proteins of $A$. cellulolyticus are more enriched with such structural and unknown modules than those of C. thermocellum (Table 2).

Many of the GH or CE catalytic modules in the multimodular proteins are associated with CBMs. In the case of a non-cellulosomal protein, a CBM may serve to deliver the parent catalytic module to a preferred site on the polysaccharide substrate.
Otherwise, an appended CBM may serve to modulate directly the hydrolytic properties of the catalytic module. Table 3 shows the number and distribution of such proteins in the genomes of both bacteria, A. cellulolyticus and $C$. thermocellum. Interestingly, 38 of the dockerin-containing enzymes in A. cellulolyticus consist of both a catalytic module and a CBM, most of the latter mostly families 3 and 6 (Table 3). In addition, another 12 non- cellulosomal enzymes contain an appended CBM. Although A. cellulolyticus contains approximately double the number of dockerincontaining proteins as $C$. thermocellum, the two species have the same number of CBM-appended enzymes (Table 2), and their distribution into different CAZy families largely overlaps.

Even more intriguing are the 10 multi-functional enzymes of $A$. cellulolyticus, which harbor a combination of at least two catalytic modules, including one or two GHs, CEs, PLs and/or glycosyl transferases (GTs), on the same polypeptide (Table 4). In A. cellulolyticus, some of these enzymes do not contain a dockerin module. In contrast, $C$. thermocellum codes for 8 multi-functional dockerin-containing enzymes, and Ruminococcus flavefaciens FD-1 codes for 18 dockerin-containing multi-functional enzymes. As stated in an earlier section, both genomes encode for two GH48 enzymes - one cellulosomal and another non-cellulosomal. In C. thermocellum, there are two separate non-cellulosomal enzymes - Cel48Y (GH48CBM3b) and the other Cel9I (GH9-CBM3c-CBM3b), whereas in $A$. cellulolyticus the two catalytic modules are fused together into a single polypeptide chain that share a single cellulose-binding CBM3b, thus forming a multifunctional non-cellulosomal enzyme (GH48-GH9CBM3c-CBM3b, [GenBank:ZP_09464448]).

\section{Putative cellulosome-related regulatory elements}

It is clear that such an elaborate cellulosome system in A. cellulolyticus would require a regulatory mechanism by which the bacterium controls expression of its cellulosomal genes. One possible regulator may be inherent in the two types of cohesin modules (i.e., type I and type II), which, like in C. thermocellum, signifies

Table 2 Summary of protein modules in cellulosomal proteins

\begin{tabular}{|c|c|c|c|c|c|c|c|}
\hline & \multirow[t]{2}{*}{ Cohesins } & \multirow{2}{*}{$\begin{array}{l}\text { Dockerin- } \\
\text { containing } \\
\text { proteins }^{\mathrm{a}}\end{array}$} & \multicolumn{5}{|c|}{ Modules in dockerin-containing proteins } \\
\hline & & & Catalytic modules ${ }^{b}$ & Structural modules ${ }^{c}$ & $\mathrm{CBMs}^{\mathrm{b}}$ & Cohesins & Others $^{\mathrm{d}}$ \\
\hline A. cellulolyticus & 41 & $143(5)$ & 74 & 46 & 53 & 13 & 36 \\
\hline C. thermocellum & 29 & $73(3)$ & 51 & 25 & 47 & 9 & 3 \\
\hline
\end{tabular}

${ }^{a}$ Numbers in parenthesis indicate the number of $\mathrm{X} 60$-dockerin modular pairs in the given species.

${ }^{\text {b }}$ Catalytic modules, such as: GH, PL, CE, and CBMs according to CAZy (http://www.cazy.org/).

c Structural domains are defined in Pfam (http://pfam.sanger.ac.uk/), such as: FN3, GDSL, SNGH, CotH, TRX-like, Kelch-like, SLH, RshA, LRR, PKD.

d Others include Pfam domains such as peptidases, serpins, DUF303, DUF1565, DUF3237. 
Table 3 Genome-wide co-occurrence of CBMs together with either GH or CE modules in A. cellulolyticus vs. C.

\begin{tabular}{|c|c|c|c|c|c|c|c|c|c|c|c|c|c|c|c|c|c|c|c|}
\hline CBM families & 3 & 4 & 6 & 9 & 11 & 13 & 22 & 23 & 27 & 30 & 32 & 34 & 35 & 42 & 44 & 48 & 50 & 54 & 62 \\
\hline GH 5 & $3 / 1$ & & $2 / 0$ & & $0 / 1$ & $1 / 0$ & & & & & $1 / 1$ & & & & & & & & $1 / 0$ \\
\hline GH 9 & 19/10 & $0 / 2$ & & & & & & & & 0/1 & & & & & $1 / 0$ & & & & \\
\hline GH 10 & & & $2 / 1$ & $2 / 2$ & & & $4 / 5$ & & & & & & & & & & & & \\
\hline GH 11 & & & $1 / 1$ & & & & & & & & & & & & & & & & \\
\hline GH 13 & & & & & & & & & & & & 0/1 & & & & $2 / 1$ & & & \\
\hline GH 16 & & $0 / 4$ & & & & & & & & & & & & & & & & $0 / 1$ & \\
\hline GH 18 & $1 / 0$ & & & & & & & & & & & & & & & & $2 / 2$ & & \\
\hline GH 26 & & & & & $0 / 1$ & & & $1 / 0$ & $1 / 0$ & & & & $3 / 2$ & & & & & & \\
\hline GH 30 & & & $1 / 1$ & & & & & & & & & & & $0 / 1$ & & & & & \\
\hline GH 39 & & & & & & & & & & & & & $0 / 2$ & & & & & & \\
\hline GH 43 & & & $2 / 3$ & & & $1 / 1$ & & & & & & & & $0 / 3$ & & & & & \\
\hline GH 44 & & & & & & & & & & $0 / 1$ & & & & & $1 / 1$ & & & & \\
\hline GH 48 & $1 / 1$ & & & & & & & & & & & & & & & & & & \\
\hline CE 1 & & & $2 / 1$ & & & & & & & & & & & & & & & & \\
\hline CE 4 & & & $1 / 1$ & & & & & & & & & & & & & & & & \\
\hline CE 6 & & & $1 / 0$ & & & $1 / 0$ & & & & & & & & & & & & & $1 / 0$ \\
\hline CE 12 & & & & & & & & & & & & & $4 / 2$ & & & & & & \\
\hline
\end{tabular}

The number of proteins with the combination of the specified modules is noted in the genomes of A. cellulolyticus (left) vs. C. thermocellum (right).

at least two divergent specificities of cohesin-dockerin interaction in this species.

Recently, a distinctive system of cellulosome gene regulation was proposed. A carbohydrate-sensing mechanism was described in C. thermocellum [48-50], suggesting that a set of putative $\sigma$ and anti- $\sigma$ factors are activated by extracellular polysaccharides. Thus, the different components of the cellulosic biomass would be detected extracellularly by corresponding RsgI-borne binding elements (CBMs, GHs, etc.), and appropriate signals are transmitted intracellularly. This in turn was proposed to disassociate the interaction between the intracellular portions of the RsgIlike proteins and complementary $\sigma^{\mathrm{I}}$-like factors, resulting in the release of the $\sigma^{\mathrm{I}} \mathrm{s}$, followed by their association with RNA polymerase and transcription of corresponding genes involved in cellulose utilization. Interestingly, analysis of the other known cellulosome-producing bacterial genomes (e.g., C. cellulolyticum and C. cellulovorans) revealed only a single RsgI-like protein, which lacks a recognizable $\mathrm{C}$-terminal binding element. It therefore appeared that an extensive RsgImediated carbohydrate-sensing mechanism is restricted to C. thermocellum.

It was thus of interest to evaluate the status of the RsgI-like proteins in A. cellulolyticus. Indeed, analysis of the genome revealed multiple copies of genes coding for $\sigma^{\mathrm{I}}$-like factors and their cognate membrane-associated
RsgI-like (anti- $\sigma^{\mathrm{I}}$ ) factors, which may be involved in regulatory mechanisms of cellulosomal and related cellulase genes. Twelve putative $\sigma^{\mathrm{I}} /$ RsgI-like proteins were detected in the $A$. cellulolyticus genome (Table 5), as opposed to the eight in C. thermocellum. The A. cellulolyticus RsgI- like proteins contain predicted C-terminal modules such as CBM3, CBM42, CBM35, PA14-like, but none appeared to contain a $\mathrm{GH}$ module like the ones detected in C. thermocellum [50]. Significantly, most of the putative $\sigma^{\mathrm{I}}$-like proteins of $A$. cellulolyticus have orthologs in C. thermocellum, some of which have been validated experimentally.

For example, the ability of $\sigma^{\mathrm{I1}}$ of $C$. thermocellum to activate the promoters of sigI1 and a family 48 cellulase, celS, was demonstrated in vitro [49]. In addition, the CBMs were shown to bind selectively to typical plant cell wall polysaccharides [48]. Interestingly, genes encoding the $\sigma^{\mathrm{I}} /$ RsgI regulatory systems are often found in genomic loci, where they are associated with other genes encoding dockerin- and cohesin-containing proteins (e.g., celE, cel124, cel8A, scaF etc.).

The multiple regulatory factors which we identified in A. cellulolyticus thus mirror the extensive regulatory system described previously in $C$. thermocellum, and may control the expression levels of cellulosomal and noncellulosomal genes to reflect changes in the plant cellwall substrates during the process of decomposition. Moreover, some of these factors may govern processes 
Table 4 Multifunctional proteins in A. cellulolyticus vs. C. thermocellum

\begin{tabular}{|c|c|c|c|}
\hline \multicolumn{2}{|c|}{ A. cellulolyticus } & \multicolumn{2}{|l|}{ C. thermocellum } \\
\hline \multicolumn{4}{|c|}{ A. Homologous cellulosomal enzymes } \\
\hline GH11-CBM6-Doc-CE4 & ZP_09464944 & GH11-CBM6-DoC-CE4 & Cthe_2972;XynA/U \\
\hline PL1-Doc-PL9 & ZP_09465691 & PL1-Doc-CBM35-PL9 & Cthe_2179 \\
\hline CE12-DOC-CBM35-CE12 & ZP_09463564 & CE12-DOC-CBM35-CE12 & Cthe_3141 \\
\hline CE12-Doc-CBM35-CE12 & ZP_09465667 & & \\
\hline \multicolumn{4}{|c|}{ B. Non-homologous cellulosomal enzymes } \\
\hline GH5-CBM6-CBM13-CBM62-Doc-CE6 & ZP_09463297 & CBM30-GH9-GH44-Doc-CBM44 & Cthe_0624;CelJ \\
\hline GH5-Doc-CE2 & ZP_09464730 & CBM22-GH10-CBM22-Doc-CE1 & Cthe_0912;XynY \\
\hline \multirow[t]{3}{*}{ CE1-CBM6-Doc-GH10 } & ZP_09465552 & GH26-GH5-CBM11-Doc & Cthe_1472;CelH \\
\hline & & GH30-CBM42-GH43-Doc & Cthe_2139 \\
\hline & & CE3-CE3-DOC & Cthe_0798 \\
\hline \multicolumn{4}{|l|}{ C. Non-cellulosomal enzymes } \\
\hline GH48-GH9-CBM3c-CBM3b & ZP_09464448 & & \\
\hline GH18-CE4-GT2 & ZP_09465738 & & \\
\hline GT84-GH94 & ZP_09462312 & & \\
\hline
\end{tabular}

Domain architectures and their corresponding accession numbers or names are listed above. Catalytic modules are marked in bold. GH, glycoside hydrolase; $\mathrm{PL}$, polysaccharide lyase; $\mathrm{CE}$, carbohydrate esterase; $\mathrm{CBM}$, carbohydrate-binding module; $\mathrm{GT}$, glycosyl transferase; Doc, dockerin; numbers indicate family of the indicated module.

in the bacterium, which are not directly involved in plant cell wall degradation.

\section{Conclusions}

Early electron microscopy observations of $A$. cellulolyticus demonstrated its particularly elaborate cell surface ultrastructure and its cellulose-degrading activities $[16,51]$. The availability of its genome sequence has enabled a better appreciation of the complex and modular nature of its cellulosome. Compared to $C$. thermocellum, the cellulosomal architecture of $A$. cellulolyticus is more extensive, encoding twice the number of cohesin- and dockerin-containing proteins, with previously undescribed combinations of protein modules. Yet, certain elements of the basic structural scaffoldins, which dictate the assembly of the various functional carbohydrate-degrading enzymes, are maintained in both species. In addition, both species exhibit elaborate cell-anchoring and gene-regulation systems. Interestingly, the multiplicity of $\sigma^{\mathrm{I}} /$ RsgI-like proteins may be characteristic of cellulosome-producing bacteria that contain multiple- scaffoldin gene clusters, like A. cellulolyticus and $C$. thermocellum, as opposed to those like $C$. cellulolyticum, that contain enzyme-linked gene clusters.

This work provides a blueprint for understanding the cellulosome system of this intriguing cellulose-degrading bacterium and paves the way for studying the specific role of its cellulosomal protein components in the degradation of plant cell-wall carbohydrates. It is clear that the bacterium utilizes a sophisticated system for efficient hydrolysis of crystalline cellulose of the plant cell wall. The cohesin-containing proteins of $A$. cellulolyticus present a broader diversity and modularity than those of C. thermocellum, where cohesins are associated in unconventional modular combinations, and their functional roles are yet to be defined.

\section{Methods}

Genomes source

Draft genome sequences of Acetivibrio cellulolyticus CD2 (DSM 1870, ATCC 33288) (30 Dec. 2011), and Clostridium thermocellum ATCC 27405 (16 Feb. 2007) were obtained from GenBank (accession: AEDB00000000 and CP000568, respectively). Assembly of $A$. cellulolyticus genome was approached by a combination of sequencing methods, using Sanger, 454-Titanium, 454 Titanium Paired-end and Solexa Paired-end technologies, as detailed in Hemme et al. [22]. The genome assembled into 112 contigs with an average coverage depth of x71.9+/- 6.3 (interval of depths $9-111$ ). Protocols of the A. cellulolyticus sequencing methods, assemblies and annotation are detailed in Land et al. [52].

\section{Sequence identification of cohesins and dockerins}

BLAST [53] searches were applied on A. cellulolyticus DNA contigs and predicted proteins, using sequences of known cohesin and dockerin modules as queries. All hits above E-value of 10-4 were retrieved and inspected individually, by examining their characteristic sequence 
Table 5 Putative $\sigma^{\prime}$ and anti- $\sigma^{\prime}$ regulatory factors in Acetivibrio cellulolyticus CD2

\begin{tabular}{llll}
\hline sigl-like gene & rsgl-like pair & C-terminal sensing domain & Ortholog in C. thermocellum \\
\hline ZP_09464729 & ZP_09464728 & CBM3 & Cthe_0403 \\
ZP_09464331 & ZP_09464330 & CBM3 & Cthe_0058 \\
ZP_09466014 & ZP_09466013 & CBM3 & Cthe_0268 \\
ZP_09463653 & ZP_09463652 & CBM3 & Cthe_0058 \\
ZP_09461804 & ZP_09461805 & CBM42 & Cthe_1272 \\
ZP_09463236 & ZP_09463235 & PA14, CBM35 & Cthe_0315 \\
ZP_09464238 & ZP_09464237 & PA14,PA14 & Cthe_1272 \\
ZP_09464575 & ZP_09464574 & unknown & Cthe_0403 \\
ZP_09464240 & ZP_09464239 & S1/S6 peptidase & Cthe_0058 \\
ZP_09463889 & ZP_09463888 & unknown & Cthe_2521 \\
ZP_09466630 & ZP_09466631 & unknown & Cthe_2974 \\
ZP_09465751 & ZP_09465752 & unknown & Cthe_2974
\end{tabular}

features. Obvious dockerin modules were expected to contain two $\mathrm{Ca}+2$-binding repeats, putative helices and linker regions. Low-scoring hits of dockerins and cohesins were examined by comparing them against known dockerin or cohesin sequences, respectively. Sequence logos of dockerins were created with Weblogo v.2.8.2 (http://weblogo.berkeley.edu/) [54]. Multiple sequence alignment was obtained using CLASTALW [55], with manual corrections when needed.

The scaffoldin genes from A. cellulolyticus ATCC 33288 which were manually sequenced [17-19] are ScaA, [GenBank: AF155197]; ScaB, [GenBank: AY221112]; ScaC, [GenBank: AY221113], ScaD, [GenBank: AY221114]). The cohesin dendrogram was generated using PhyML algorithms (with LG substitution model, and default parameters of the Approximate Likelihood-Ratio test) [56] and visualized using TreeView [57].

\section{Annotation of dockerin-containing enzymes}

Dockerin-containing proteins of $A$. cellulolyticus CD2 and $C$. thermocellum ATCC 27405 were annotated by CAZy database (http://www.cazy.org) [41], in order to bioinformatically analyze their catalytic modules. This includes identification of the catalytic modules and their classification into family types, according to sequence conservation, for glycoside hydrolases, carbohydrate esterases, polysaccharide lyases, carbohydrate-binding modules and glycosyl transferases. Additional conserved domains of the proteins were analyzed using the CDsearch website (http://www.ncbi.nlm.nih.gov/Structure/ cdd/wrpsb.cgi) and the Pfam database (http://pfam.sanger.ac.uk/). Putative cellulosome-related regulatory elements were identified by BLAST searches and sequence similarity using known elements from C. thermocellum as queries [48-50].

\section{Additional file}

Additional file 1: Table S1. Cellulosomal and non-cellulosomal CAZyme proteins in A. cellulolyticus. The modular architecture of the indicated proteins show only the CAZy-related modules: GH, glycoside hydrolase; $\mathrm{PL}$, polysaccharide lyase; $\mathrm{CE}$, carbohydrate esterase; CBM, carbohydrate-binding module; Doc, dockerin; Coh, cohesin, SLH, S-layer homology modules. Numbers indicate family of the indicated module. A. Cohesin-containing proteins. B. Dockerin-containing proteins. C. Non-cellulosomal CAZymes

\section{Abbreviations}

CAZy: Carbohydrate-active enzymes; GH: Glycoside hydrolase; CE: Carbohydrate esterase; PL: Polysaccharide lyase; CBM: Carbohydratebinding module; GT: Glycosyl transferases; CBM: Carbohydrate-binding module; SLH: S-layer homology; X-doc: X-dockerin; Sca: Scaffoldin.

\section{Competing interests}

The authors declare that they have no competing interests.

\section{Acknowledgements}

We thank Yuval Hamberg for helpful discussions. Parts of this research were supported by grants from the Israel Science Foundation (Grant nos 966/09, 159/07 and 24/11), by The Israeli Centers of Research Excellence (I-CORE) program, (Center No. 152/11), by The Alternative Energy Research Initiative Bioenergy Consortium at the Weizmann Institute of Science and the ChinaIsrael Scientific Research Cooperation. E.A.B. is the incumbent of The Maynard I. and Elaine Wishner Chair of Bio-organic Chemistry.

\section{Author details}

'Department of Biological Chemistry, The Weizmann Institute of Science, Rehovot, Israel. ${ }^{2}$ Department of Molecular Microbiology and Biotechnology, Tel Aviv University, Ramat Aviv, Tel Aviv, Israel. ${ }^{3}$ Architecture et Fonction des Macromolecules Biologiques, CNRS and Universite Aix-Marseilles I \& II, Marseilles, France. ${ }^{4}$ Department of Botany and Microbiology, and Institute for Environmental Genomics, University of Oklahoma, Norman, OK, USA.

\section{Authors' contributions}

$\mathrm{BD}$ and $\mathrm{EAB}$ conceived of the project and wrote the manuscript. BD, $\mathrm{IB}, \mathrm{BH}$ and $\mathrm{PC}$ analyzed the genome data. CLH, YH and JZ sequenced the genome. $B D, R L$ and $E A B$ wrote the paper. All authors read and approved the final manuscript. 
Received: 21 February 2012 Accepted: 30 May 2012

Published: 30 May 2012

\section{References}

1. Lynd LR, Laser MS, Bransby D, Dale BE, Davison B, Hamilton R, Himmel M, Keller M, McMillan JD, Sheehan J, et al: How biotech can transform biofuels. Nature Biotechnol 2008, 26:169-172

2. Himmel ME, Xu Q, Luo Y, Ding S-Y, Lamed R, Bayer EA: Microbial enzyme systems for biomass conversion: Emerging paradigms. Biofuels 2010, 1:323-341

3. Bayer EA, Chanzy H, Lamed R, Shoham Y: Cellulose, cellulases and cellulosomes. Curr Opin Struct Biol 1998, 8:548-557.

4. Bayer EA, Belaich J-P, Shoham Y, Lamed R: The cellulosomes: Multi-enzyme machines for degradation of plant cell wall polysaccharides. Annu Rev Microbiol 2004, 58:521-554.

5. Bayer EA, Lamed R, White BA, Flint HJ: From cellulosomes to cellulosomics. Chem Rec 2008, 8:364-377.

6. Demain AL, Newcomb M, Wu JH: Cellulase, clostridia, and ethanol. Microbiol Mol Biol Rev 2005, 69(1):124-154.

7. Doi RH, Kosugi A: Cellulosomes: plant-cell-wall-degrading enzyme complexes. Nat Rev Microbiol 2004, 2(7):541-551.

8. Bayer EA, Shimon LJW, Lamed R, Shoham Y: Cellulosomes: structure and ultrastructure. J Struct Biol 1998, 124:221-234.

9. Bayer EA, Shoham Y, Lamed R: Cellulose-decomposing prokaryotes and their enzyme systems. In In: The Prokaryotes, Third Edition. Edited by Dworkin M, Falkow S, Rosenberg E, Schleifer K-H, Stackebrandt E. New York: Springer; 2006:578-617. vol. 2

10. Raman B, Pan C, Hurst GB, Rodriguez M, McKeown CK, Lankford PK, Samatova NF, Mielenz JR: Impact of pretreated switchgrass and biomass carbohydrates on Clostridium thermocellum ATCC 27405 cellulosome composition: a quantitative proteomic analysis. PLoS One 2009, 4:e5271.

11. Schwarz WH, Zverlov W, Bahl H: Extracellular glycosyl hydrolases from Clostridia. Advan Appl Microbiol 2004, 56:215-261.

12. Khan AW: Cellulolytic enzyme system of Acetivibrio cellulolyticus, a newly isolated anaerobe. J Gen Microbiol 1980, 121:499-502.

13. Patel GB, Khan AW, Agnew BJ, Colvin JR: Isolation and characterization of an anaerobic cellulolytic microorganism, Acetivibrio cellulolyticus, gen. nov., sp. nov. Int J Syst Bacteriol 1980, 30:179-185.

14. Saddler JN, Khan AW: Cellulase production by Acetivibrio cellulolyticus. Can J Microbiol 1980, 26:760-765.

15. Saddler JN, Khan AW: Cellulolytic enzyme system of Acetivibrio cellulolyticus. Can J Microbiol 1981, 27.288-294.

16. Lamed R, Naimark J, Morgenstern E, Bayer EA: Specialized cell surface structures in cellulolytic bacteria. J Bacteriol 1987, 169:3792-3800.

17. Ding S-Y, Bayer EA, Steiner D, Shoham Y, Lamed R: A novel cellulosomal scaffoldin from Acetivibrio cellulolyticus that contains a family- 9 glycosyl hydrolase. J Bacteriol 1999, 181:6720-6729.

18. Xu Q, Gao W, Ding S-Y, Kenig R, Shoham Y, Bayer EA, Lamed R: The cellulosome system of Acetivibrio cellulolyticus includes a novel type of adaptor protein and a cell-surface anchoring protein. J Bacterio/ 2003, 185:4548-4557.

19. Xu Q, Barak Y, Kenig R, Shoham Y, Bayer EA, Lamed R: A novel Acetivibrio cellulolyticus anchoring scaffoldin that bears divergent cohesins. Bacteriol 2004, 186:5782-5789.

20. Noach I, Alber O, Bayer EA, Lamed R, Levy-Assaraf M, Shimon LJW, Frolow F: Crystallization and preliminary X-ray analysis of Acetivibrio cellulolyticus cellulosomal type II cohesin module: Two versions having different linker lengths. Acta Cryst 2008, F64:58-61.

21. Noach I, Frolow F, Alber O, Lamed R, Shimon LJW, Bayer EA: Inter-modular linker flexibility revealed from crystal structures of adjacent cellulosomal cohesins of Acetivibrio cellulolyticus. J Mol Biol 2009, 391:86-97.

22. Hemme CL, Mouttaki H, Lee YJ, Zhang G, Goodwin L, Lucas S, Copeland A, Lapidus A, Glavina del Rio T, Tice H, et al: Sequencing of multiple clostridial genomes related to biomass conversion and biofuel production. J Bacteriol 2010, 192(24):6494-6496.

23. Blouzard JC, Coutinho PM, Fierobe HP, Henrissat B, Lignon S, Tardif C, Pagès $\mathrm{S}$, de Philip P: Modulation of cellulosome composition in Clostridium cellulolyticum: adaptation to the polysaccharide environment revealed by proteomic and carbohydrate-active enzyme analyses. Proteomics 2010, 10:541-554
24. Brown SD, Raman B, McKeown CK, Kale SP, He ZL, Mielenz JR: Construction and evaluation of a Clostridium thermocellum ATCC 27405 wholegenome oligonucleotide microarray. Appl Biochem Biotechnol 2007 , 137:663-674.

25. Tamaru Y, Miyake H, Kuroda K, Nakanishi A, Kawade Y, Yamamoto K, Uemura M, Fujita Y, Doi RH, Ueda M: Genome sequence of the cellulosome-producing mesophilic organism Clostridium cellulovorans 743B. J Bacteriol 2010, 192:901-902.

26. Berg Miller ME, Antonopoulos DA, Rincon MT, Band M, Bari A, Akaikol T, Hernandez A, Kim R, Liu L, Thimmapuram J, et al: Diversity and strain specificity of plant cell wall degrading enzymes revealed by the draft genome of Ruminococcus flavefaciens FD-1. PLoS One 2009, 4:e6650.

27. Chauvaux S, Matuschek M, Béguin P: Distinct affinity of binding sites for $\mathrm{S}$ - layer homologous domains in Clostridium thermocellum and Bacillus anthracis cell envelopes. J Bacteriol 1999, 181:2455-2458.

28. Lemaire $M$, Ohayon $H$, Gounon P, Fujino T, Béguin P: OlpB, a new outer layer protein of Clostridium thermocellum, and binding of its S-layerlike domains to components of the cell envelope. J Bacteriol 1995 177:2451-2459.

29. Lin C, Urbance JW, Stahl DA: Acetivibrio cellulolyticus and Bacteroides cellulosolvens are members of the greater clostridial assemblage. FEMS Microbiol Lett 1994, 124:151-155.

30. Pinheiro BA, Gilbert HJ, Sakka K, Fernandes VO, Prates JA, Alves VD, Bolam DN, Ferreira LM, Fontes CM: Functional insights into the role of novel type I cohesin and dockerin domains from Clostridium thermocellum. Biochem J 2009, 424(3):375-384.

31. Fendri I, Tardif $C$, Fierobe HP, Lignon S, Valette $O$, Pagès $S$, Perret $S$ : The cellulosomes from Clostridium cellulolyticum: identification of new components and synergies between complexes. FEBS Journal 2009, 276:3076-3086.: The cellulosomes from Clostridium cellulolyticum: identification of new components and synergies between complexes. FEBS J 2009, 276:3076-3086.

32. Bayer EA, Henrissat B, Lamed R: The cellulosome: A natural bacterial strategy to combat biomass recalcitrance. In In: Biomass Recalcitrance. Edited by Himmel ME. London: Blackwell; 2008:407-426.

33. Rincon MT, Dassa B, Flint HJ, Travis AR, Jindou S, Borovok I, Lamed R, Bayer EA, Henrissat B, Coutinho PM, et al: Abundance and diversity of dockerincontaining proteins in the fiber-degrading rumen bacterium, Ruminococcus flavefaciens FD1. PLoS One 2010, 5:e12476.

34. Adams JJ, Jang CJ, Spencer HL, Elliott M, Smith SP: Expression, purification and structural characterization of the scaffoldin hydrophilic X-module from the cellulosome of Clostridium thermocellum. Protein Expr Purif 2004, 38(2):258-263

35. Pagès $S$, Belaich A, Belaich J-P, Morag E, Lamed R, Shoham $Y$, Bayer EA: Species-specificity of the cohesin-dockerin interaction between Clostridium thermocellum and Clostridium cellulolyticum: Prediction of specificity determinants of the dockerin domain. Proteins 1997, 29:517-527.

36. Mechaly A, Yaron S, Lamed R, Fierobe H-P, Belaich A, Belaich J-P, Shoham Y, Bayer EA: Cohesin-dockerin recognition in cellulosome assembly: Experiment versus hypothesis. Proteins 2000, 39:170-177.

37. Mechaly A, Fierobe H-P, Belaich A, Belaich J-P, Lamed R, Shoham Y, Bayer EA: Cohesin-dockerin interaction in cellulosome assembly: A single hydroxyl group of a dockerin domain distinguishes between non-recognition and high- affinity recognition. J Biol Chem 2001, 276:9883-9888. and Erratum 19678.

38. Khan AW, Meek E, Sowden LC, Colvin JR: Emendation of the genus Acetivibrio and description of Acetivibrio cellulosolvens sp. nov., a nonmotile cellulolytic mesophile. Int J Syst Bacterio/ 1984, 34:419-422

39. Sanchez CR, Peres CS, Barbosa HR: Growth and endoglucanase activity of Acetivibrio cellulolyticus grown in three different cellulosic substrates. Rev Microbiol 1999, 30:310-314.

40. Murray WD: Acetivibrio cellulosolvens is a synonym for Acetivibrio cellulolyticus: Emendation of the genus Acetivibrio. Int J Syst Bacteriol 1986, 36:314-316.

41. Cantarel BL, Coutinho PM, Rancurel C, Bernard T, Lombard V, Henrissat B: The Carbohydrate-Active Enzymes database (CAZy): an expert resource for glycogenomics. Nucl Acids Res 2009, 37:D233-238.

42. Gal L, Gaudin C, Belaich A, Pagès S, Tardif C, Belaich J-P: CelG from Clostridium cellulolyticum: a multidomain endoglucanase acting efficiently on crystalline cellulose. J Bacteriol 1997, 179:6595-6601. 
43. Irwin D, Shin D-H, Zhang S, Barr BK, Sakon J, Karplus PA, Wilson DB: Roles of the catalytic domain and two cellulose binding domains of Thermomonospora fusca E4 in cellulose hydrolysis. J Bacterio/ 1998, 180:1709-1714.

44. Sakon J, Irwin D, Wilson DB, Karplus PA: Structure and mechanism of endo/exocellulase E4 from Thermomonospora fusca. Nature Struct Biol 1997, 4:810-818

45. Gilad R, Rabinovich L, Yaron S, Bayer EA, Lamed R, Gilbert HJ, Shoham Y: Cell, a non-cellulosomal family-9 enzyme from Clostridium thermocellum, is a processive endoglucanase that degrades crystalline cellulose. J Bacteriol 2003, 185:391-398.

46. Jindou S, Xu Q, Kenig R, Shoham Y, Bayer EA, Lamed R: Novel architectural theme of family- 9 glycoside hydrolases identified in cellulosomal enzymes of Acetivibrio cellulolyticus and Clostridium thermocellum. FEMS Microbiol Lett 2006, 254:308-316.

47. Brás JL, Cartmell A, Carvalho AL, Verzé G, Bayer EA, Vazana Y, Correia MA, Prates JA, Ratnaparkhe $S$, Boraston $A B$, et al: Structural insights into a unique cellulase fold and mechanism of cellulose hydrolysis. Proc Natl Acad Sci USA 2011, 108:5237-5242.

48. Kahel-Raifer H, Jindou S, Bahari L, Nataf $Y$, Shoham Y, Bayer EA, Borovok I, Lamed $R$ : The unique set of putative membrane-associated anti- $\sigma$ factors in Clostridium thermocellum suggests a novel extracellular carbohydratesensing mechanism involved in gene regulation. FEMS Microbiol Lett 2010, 308:84-93.

49. Nataf Y, Bahari L, Kahel-Raifer H, Borovok I, Lamed R, Bayer EA, Sonenshein $A L$, Shoham Y: Clostridium thermocellum cellulosomal genes are regulated by extracytoplasmic polysaccharides via alternate sigma factors. Proc Natl Acad Sci USA 2010, 10718646-51:18646-18651.

50. Bahari L, Gilad Y, Borovok I, Dassa B, Kahel-Raifer H, Jindou S, Nataf Y, Shoham Y, Lamed R, Bayer EA: Glycoside hydrolases as components of putative carbohydrate biosensor proteins in Clostridium thermocellum. $J$ Ind Microbiol Biotechnol 2011, 38:825-832.

51. Lamed R, Naimark J, Morgenstern E, Bayer EA: Scanning electron microscopic delineation of bacterial surface topology using cationized ferritin. J Microbiol Methods 1987, 7:233-240.

52. Land M, Pukall R, Abt B, Goker M, Rohde M, Glavina Del Rio T, Tice H, Copeland A, Cheng JF, Lucas S, et al: Complete genome sequence of Beutenbergia cavernae type strain (HKI 0122). Stand Genomic Sci 2009, 1(1):21-28.

53. Altschul SF, Madden TL, Schäffer AA, Zhang J, Zhang Z, Miller W, Lipman DJ: Gapped BLAST and PSI-BLAST: a new generation of protein database search programs. Nucl Acids Res 1997, 25:3389-3402.

54. Crooks GE, Hon G, Chandonia JM, Brenner SE: WebLogo: A sequence logo generator. Genome Res 2004, 14:1188-1190.

55. Higgins D, Thompson J, Gibson T, Thompson JD, Higgins DG, Gibson TJ: CLUSTAL W: improving the sensitivity of progressive multiple sequence alignment through sequence weighting, position-specific gap penalties and weight matrix choice. Nucleic Acids Res 1994, 22:4673-4680.

56. Guindon S, Dufayard JF, Lefort V, Anisimova M, Hordijk W, Gascuel O: New algorithms and methods to estimate maximum-likelihood phylogenies: Assessing the performance of PhyML 3.0. Syst Biol 2010, 59:307-321.

57. Page RD: TreeView: an application to display phylogenetic trees on personal computers. Comput Appl Biosci 1996, 12:357-358.

doi:10.1186/1471-2164-13-210

Cite this article as: Dassa et al:: Genome-wide analysis of acetivibrio cellulolyticus provides a blueprint of an elaborate cellulosome system. BMC Genomics 2012 13:210

\section{Submit your next manuscript to BioMed Central and take full advantage of:}

- Convenient online submission

- Thorough peer review

- No space constraints or color figure charges

- Immediate publication on acceptance

- Inclusion in PubMed, CAS, Scopus and Google Scholar

- Research which is freely available for redistribution 